\title{
Eclipse of the free quark charge
}

\section{The existence of electric charges which are fractions of the elementary electronic charge has been widely canvassed, but has now been cast in doubt. The search has however been a spur to ingenious experiment.}

SINCE the demonstration by Robert Millikan that the electric charge acquired by a droplet suspended against the force of gravity by an electrostatic field is invariably a multiple of some constant amount, the same technique has been variously held up as a demonstration of the precision of physical measurements and as proof of how important natural constants can be determined directly. Only in recent years has the complaint that Millikan may have improved on nature in presenting his results been considered seriously, and then only in the spirit that there have accumulated so many other pieces of evidence bearing on the constancy of the unit charge, that the elegance of Millikan's experiment remains a model for the rest of us.

This was true at least until the hunt began for the particles called quarks which, when their existence was first predicted by Murray Gell-Mann, were known to have only one distinctive property - a fractional electric charge, either one-third that of an electron or twice as much, plus or minus. That, it will be recalled, was the period when there was such uncertainty about the masses of the quarks that schemes for hunting them with vacuum cleaners beneath electric fences, or dredging for them on the sea-bottom, were all the rage.

William Fairbank from Stanford University had a better idea. He set out to repeat Millikan's measurement but with extra ingenuity and with much greater precision. Fairbank's announcement, nearly a decade ago, that small spheres of niobium suspended by magnetic levitation appear to behave in electric fields as if they carried fractional electric charges seemed, at the time, as neat a pointer to the existence of quarks as there could be. Only as the lesson has sunk home that unbound quarks do not exist has his elegant experiment been counted as a challenge. For if Fairbank's apparently fractional charges cannot be free quarks, what are they?

The consequence has been a prolonged and detailed examination of the sideeffects that may vitiate the interpretation of Fairbank's measurement. All kinds of sources of sytematic error have been examined. A vivid measure of the exhaustive way in which the issue has been tackled is that one of the most serious sources of error may be the variation from place to place of the electrical properties of the surface of a metallic object. Specifically, there may be patches on a metallic surface whose pro- perties differ from the remainder, perhaps because the crystal structure in the locality enables electrons to be detached with a voltage a little smaller or larger than the average work function.

Variations of the work function of a few tens of millivolts are well documented. Their consequences for measurements in the Fairbank mould are obviously important. If, for example, it is planned to drive a suspended charged object in one direction or another by means of a uniform electric field generated by applying some voltage to a pair of parallel metallic plates, patches of abnormal work function will undermine the assumption that each surface is an equipotential surface and thus that the electric field between them is uniform. So how can unavoidable difficulties such as these be avoided?

This is the challenge to which have risen D. Liebowitz, M. Binder and K.O.H. Ziock from the University of Virginia at Charlottesville. In Physical Review Letters on 23 May $(50,1640 ; 1983)$, they describe an experiment which is in many ways simpler than others in this genre but in which variations of the surface properties of suspended spherical objects can be ironed out. This is not the first attempt to allow for departures from electrical spherical symmetry, whose consequences can spuriously endow the sphere with the appearance of extra electric charge. Attempts to swamp this effect, both at Stanford and at Charlottesville, by making suspended spheres spin rapidly have apparently failed, chiefly because of the precession thus induced in the magnetic field.

The Charlottesville group has worked with steel spheres $0.2 \mathrm{~mm}$ in diameter, gigantic compared with Fairbank's particles of niobium dust. To avoid the complications arising from precession, the group has devised an apparatus in which the spheres are suspended by a vertical magnetic field and driven vertically up or down by means of the electric field between two horizontal condenser plates. The plan is to make the steel spheres spin also about a vertical axis, so that precession generated by patches on the surface of the spheres should be negligible.

Naturally, the whole equipment is evacuated. The electric field between the condenser plates (only $2 \mathrm{~cm}$ apart) is made to oscillate twice a second, and an arrangement made for a supplementary magnetic field provided by a coil to prevent the physical oscillation of the suspended ball by means of a servo-system driven by an optical sensor. The variable current in the coil is thus a means of inferring the electrostatic force on the suspended ball and thus, if patches are irrelevant, of the net electric charge it carries. To ensure that patches are irrelevant, the steel spheres are made to spin rapidly by means of an oscillating magnetic field.

The experimental arrangement is simple as well as elegant, but its designers plaintively note the difficulty they have encountered in telling not merely how quickly their steel spheres are spinning but, by simply looking, whether they are spinning at all. This is why they scratched the surface of one of their 24 steel spheres to infer that after a few minutes, their spheres were spinning at 600,000 revolutions a minute.

The results would serve only to make Millikan even more satisfied with himself than he is said to have been in real life. It is possible to induce extra electric charge on the steel spheres, spinning or otherwise, by means of ultraviolet light. The authors of the experiment report that their spinning spheres acquire only integral electric charges, and then only after these have been deliberately induced. Specifically, they "never observed charge changes that were not an integer multiple" of some highest common factor, roughly the elementary charge. The supposed elementary charges of spheres not spinning vary about the known value of the elementary charge, in some cases by as much as a third of the electronic charge, but the same spheres spinning give elementary charges much more closely bunched about the expected value. Something like the patch effect must be at work.

Where does this leave Fairbank's data? The authors of this new measurement generously say that steel is not niobium, so that what they have done does not undermine the earlier claim that fractional charges exist. It is possible to argue that the conditions of the two experiments are so different that fractional charges were somehow excluded from the Charlottesville measurement. Whatever the truth, the onus now lies with Fairbank to eliminate the patch effect with niobium spheres. If the result should be that fractional charges seem not to exist, Fairbank will at least have had had the fun of hunting quarks for more than the past decade, and the knowledge that he has stimulated a series of ingenious experiments that otherwise would never have been done.

John Maddox 